\title{
AVALIAÇÃO DA COMPOSIÇÃO CORPORAL E DO CONSUMO ALIMENTAR DE NADADORES ADOLESCENTES
}

\author{
ASSESSMENT OF BODY COMPOSITION AND DIETARY INTAKE OF ADOLESCENT SWIMMERS \\ EVALUACIÓN DE LA COMPOSICIÓN CORPORAL Y DE LA INGESTA DE ALIMENTOS \\ DE NADADORES ADOLESCENTES
}

Aline Veroneze de Mello

(Nutricionista)

Walleska Luctke Facincani Villarim (Nutricionista)

Alisson Diego Machado

(Nutricionista)

Edilene Vitorino Olivon ${ }^{1}$

(Nutricionista)

Daniele Lima da Cruz ${ }^{1}$

(Nutricionista)

Luana Romão Nogueira'

(Nutricionista)

Marcia Nacif ${ }^{1}$

(Nutricionista)

1. Universidade Presbiteriana

Mackenzie, Centro de Ciências Biológicas e da Saúde, Nutrição, São Paulo, SP, Brasil.

\section{Correspondência:}

Universidade Presbiteriana Mackenzie - CCBS Nutrição Rua da Consolação, 930 Consolação - São Paulo, SP, Brasil - 01302-907 marcia.nacif@mackenzie.br

\section{RESUMO}

Introdução: A composição corporal e a alimentação de nadadores têm influência direta em seu desempenho esportivo. Objetivo: Avaliar a composição corporal e o consumo alimentar de nadadores adolescentes. Métodos: Estudo transversal realizado com 15 nadadores adolescentes, de ambos os sexos, de um clube de São Paulo, Brasil. Aplicou-se um questionário de identificação e houve aferição de peso, estatura, circunferências corporais e dobras cutâneas. Para a avaliação do consumo alimentar foi aplicado um recordatório de 24 horas. Resultados: A maioria dos atletas apresentou percentual de gordura corporal adequado. Houve ingestão de suplementos alimentares por todos os nadadores. Observou-se elevado consumo de proteínas e baixa ingestão de carboidratos. As maiores prevalências de inadequação de micronutrientes foram para vitamina $B_{9}$, iodo $e$ cálcio. Conclusão: Os resultados sugerem a necessidade de intervenção nutricional nesse grupo de atletas.

Palavras-chave: atletas, antropometria, consumo de alimentos.

\begin{abstract}
Introduction: Body composition and nutritional habits of swimmers have a direct influence on their sports performance. Objective: To assess body composition and dietary intake of adolescent swimmers. Method: Cross-sectional study with 15 adolescent swimmers of both genders from a club in the city of São Paulo, Brazil. An identification questionnaire was applied, registering information on weight, height, body circumferences and skinfolds. For the assessment of dietary intake, a 24-hour recall was applied. Results: Most of the athletes presented appropriate body fat percentage. All swimmers ingested dietary supplements. Protein consumption was high and carbohydrate, low. The highest prevalence of inadequate intakes of micronutrients were vitamin $B_{9}$, iodine and calcium. Conclusion: The results suggest the need for nutritional intervention in this group of athletes.
\end{abstract}

Keywords: athletes, anthropometry, food consumption.

\section{RESUMEN}

Introducción: La composición corporal y la alimentación de los nadadores tienen influencia directa en su rendimiento deportivo. Objetivo: Evaluar la composición corporal y la ingesta de alimentos de nadadores adolescentes. Métodos: Estudio transversal con 15 nadadores adolescentes de ambos sexos, en un club de São Paulo, Brasil. Fue aplicado un cuestionario de identificación y fueron evaluados peso, la altura, la circunferencia del cuerpo y los pliegues de la piel. Para la evaluación de la ingesta alimentaria se aplicó de un recordatorio de 24 horas. Resultados: La mayoría de los atletas presentó porcentaje de grasa corporal adecuada. Hubo ingesta de suplementos alimenticios por todos los nadadores. Hubo un alto consumo de proteínas y la ingesta baja en carbohidratos. Las mayores prevalencias de insuficiencia de micronutrientes fueron la vitamina B9, yodo y calcio. Conclusión: Los resultados sugieren la necesidad de intervención nutricional en este grupo de atletas.

Palabras clave: atletas, antropometría, consumo de alimentos.

\section{INTRODUÇÃO}

A natação é considerada uma modalidade desportiva ou uma forma particular de locomoção na qual o nadador tem como objetivo principal percorrer uma distância previamente estabelecida no menor tempo possível ${ }^{1}$, sendo influenciado por suas características físicas ${ }^{2}$ e pela sua alimentação e hidratação, que são indispensáveis para o desempenho de atletas ${ }^{3}$.

A avaliação da composição corporal deve ser realizada para o acompanhamento do desempenho de nadadores, sendo os métodos mais utilizados os duplamente indiretos, que são fidedignos, não-invasivos e menos onerosos, quando comparados a outros mais sofisticados 4 .

A nutrição adequada também é fundamental para o desempenho esportivo, uma vez que a natação é um esporte com grande demanda energética, no qual a ingestão adequada de nutrientes é essencial ${ }^{5}$. A reposição hídrica, quando realizada de maneira insuficiente, pode depreciar o desempenho durante o exercício, ressaltando assim a sua importância6.

A prescrição de suplementos alimentares, por sua vez, deve ser direcionada com base na adequação do consumo alimentar, com período de utilização definido, e ser pautada na reavaliação sistemática não apenas do estado nutricional, mas também do plano alimentar do atleta? 
Considerando-se que a constituição física e o estado nutricional influenciam diretamente o desempenho esportivo, o presente estudo teve por objetivo avaliar a composição corporal e o consumo alimentar de nadadores adolescentes de um clube do município de São Paulo.

\section{MÉTODOS}

Foi realizado um estudo transversal com 15 nadadores adolescentes, de ambos os sexos e com idade entre 16 e 18 anos, de um clube localizado no município de São Paulo, SP, Brasil.

A coleta de dados foi realizada após a assinatura do Termo de Consentimento Livre e Esclarecido pelos atletas ou pelos pais e/ou responsáveis, no caso dos menores de 18 anos. Seguiram-se as determinações da Resolução no 196/96, do Conselho Nacional de Saúde, sendo o projeto aprovado pelo Comitê Interno de Ética em Pesquisa da Universidade Presbiteriana Mackenzie, sob número N006/03/2013.

Inicialmente foi aplicado um questionário de identificação composto por perguntas referentes à idade, sexo, modalidade esportiva, frequência e duração da prática de atividade física, consumo de suplementos alimentares e ingestão de água.

O peso foi aferido com utilização de uma balança digital (Avanutri ${ }^{\circledR}$, modelo SE871A, Brasil) com capacidade para 150 kg e sensibilidade para 0,1 kg e a estatura foi mensurada com uma fita métrica (Lemat ${ }^{\circledR}$ ) com resolução de $1 \mathrm{~mm}$ aderida a uma parede sem rodapé, a 2 metros do solo.

As circunferências de cintura e de braço (CC e CB, respectivamente) foram aferidas com utilização de uma fita métrica inelástica e flexível (Lemat ${ }^{\circledR}$, Brasil), sendo a medida de CC realizada no ponto médio entre o último arco costal e a crista ilíaca. As dobras cutâneas de tríceps $(D C T)$, bíceps (DCB), subescapular (DCSE), torácica (DCTO), suprailíaca (DCSI), abdominal (DCA) e de panturrilha medial (DCP) foram mensuradas em triplicata, com utilização de um adipômetro (Lange $\left.{ }^{\circledR}\right)$ com precisão de $1 \mathrm{~mm}$.

Para a classificação do estado nutricional foi calculado o índice de massa corporal (IMC) por idade, avaliado pelas curvas de crescimento propostas pela World Health Organization ${ }^{8}$. O percentual de gordura corporal foi estimado pelas equações propostas por Slaughter et al. ${ }^{9}$ para os atletas com 16 e 17 anos e por Jackson e Pollock ${ }^{10}$ para os que possuíam 18 anos, sendo classificado de acordo com Deurenberg, Kusters e Smit ${ }^{11}$. Mensurou-se também o percentual com a utilização de um aparelho de bioimpedância (BIA) de membros superiores $\left(O M R O N^{\circledR}\right.$, modelo HBF-306C, Omron Health Care Estados Unidos).

Os valores de circunferência muscular do braço (CMB), área muscular do braço corrigida (AMBC) e área de gordura do braço (AGB) foram classificados por Frisancho ${ }^{12}$. O risco para o desenvolvimento de alterações metabólicas foi avaliado segundo Freedman et al ${ }^{13}$ para os atletas com 16 e 17 anos e segundo a Organização Mundial da Saúde $(\mathrm{OMS})^{14}$ para aqueles com 18 anos.

Para a avaliação do consumo alimentar foi aplicado um recordatório de 24 horas, que foi preenchido com todos os alimentos, bebidas e suplementos consumidos no dia anterior à entrevista, com auxílio do software Avanutri Online ${ }^{\circledR}$, Brasil.

A ingestão de energia e macronutrientes foi avaliada de acordo com o recomendado pela Sociedade Brasileira de Medicina do Esporte $^{15}$. A prevalência de inadequação de micronutrientes foi calculada utilizando-se os valores recomendados pelas Dietary Reference Intakes, propostas pelo Institute of Medicine ${ }^{16-21}$, por meio da fórmula $z=(E A R-m e ́ d i a) / d p$, onde EAR corresponde ao valor da estimativa da necessidade, média ao valor médio consumido pelo grupo para cada um dos micronutrientes avaliados e o $d p$ ao desvio-padrão da ingestão média. A prevalência de inadequação foi calculada pelo valor de z-escore obtido pela fórmula.

\section{Análise estatística}

As variáveis qualitativas foram apresentadas por meio da distribuição de frequências absoluta e relativa e as quantitativas em média e desvio-padrão ( $m \pm d p)$. Para a correlação entre o percentual de gordura obtido pelas dobras cutâneas e pela BIA utilizou-se o teste de Pearson, com intervalos de confiança de $95 \%$. As análises foram realizadas com auxílio do programa estatístico SPSS versão 20.0.

\section{RESULTADOS}

Participaram do estudo 15 nadadores de ambos os sexos, sendo $53,3 \%(n=8)$ do sexo masculino e $46,7 \%(n=7)$ do sexo feminino, com idade média de 17,3 $\pm 0,8$ anos (tabela 1). A modalidade mais praticada correspondeu ao nado crawl, com frequência de treino de 6 dias por semana e 5 horas diárias.

No presente estudo, utilizando-se as curvas de crescimento propostas pela Organização Mundial da Saúde ${ }^{8}$, três atletas apresentaram risco para o desenvolvimento de sobrepeso (tabela 2).

Contudo, ao ser avaliado o percentual de gordura corporal, verificou-se que os mesmos se apresentaram adequados nesses atletas, confirmado pelos dados de CMB, que apontaram que os atletas possuíam musculatura desenvolvida ou adequada para a idade e pelos valores de $A G B$, que demonstraram que os atletas não apresentavam obesidade, indicando que a relação IMC/idade proposta não deve ser utilizada como único parâmetro para a classificação do estado nutricional de atletas. Houve boa correlação entre o percentual de gordura obtido pelas dobras cutâneas e pela BIA $(r=0,789)$. O risco para o desenvolvimento de alterações metabólicas não foi identificado em nenhum dos atletas (tabela 3 e figura 1).

A ingestão de suplementos alimentares foi relatada por todos os atletas do presente estudo. O consumo médio correspondeu a 6,1 $\pm 2,9$ suplementos, sendo os mais utilizados o Endurox, Waxy Maize, Accelerade, creatina e Iso WPI (tabela 4).

Tabela 1. Distribuição das características pessoais dos atletas em estudo. São Paulo, 2013.

\begin{tabular}{c|c|c}
\hline Variável & $\mathbf{n}$ & $\%$ \\
\hline Sexo & & 53,3 \\
\hline Masculino & 8 & 46,7 \\
\hline Feminino & 7 & \\
\hline Idade (anos) & & 20,0 \\
\hline 16 & 3 & 33,3 \\
\hline 17 & 5 & 46,7 \\
\hline 18 & 7 & 13,3 \\
\hline Modalidade & & 20,0 \\
\hline Borboleta & 2 & 46,7 \\
\hline Costas & 3 & 13,3 \\
\hline Crawl & 7 & 6,7 \\
\hline Medley & 2 & 100,0 \\
\hline Peito & 1 & \\
\hline Total & 15 & \\
\hline
\end{tabular}

Tabela 2. Distribuição do estado nutricional e do percentual de gordura corporal dos atletas em estudo. São Paulo, 2013.

\begin{tabular}{c|c|c|c|c|c|c}
\hline \multirow{2}{*}{ IMC/idade } & \multicolumn{6}{|c}{ Percentual de gordura corporal } \\
\cline { 2 - 8 } & \multicolumn{2}{|c|}{ Baixa } & \multicolumn{2}{c}{ Adequada } & \multicolumn{2}{c}{ Elevada } \\
\cline { 2 - 8 } & $\mathbf{n}$ & $\%$ & $\mathbf{n}$ & $\%$ & $\mathbf{n}$ & $\%$ \\
\hline Magreza & - & - & - & - & - & - \\
\hline Eutrofia & 6 & 100,0 & 6 & 66,6 & - & - \\
\hline Risco de sobrepeso & - & - & 3 & 33,3 & - & - \\
\hline Sobrepeso & - & - & - & - & - & - \\
\hline Obesidade & - & - & - & - & - & - \\
\hline Total & 6 & 100,0 & 9 & 100,0 & - & - \\
\hline
\end{tabular}




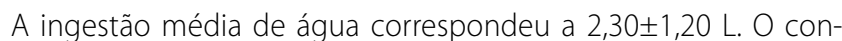
sumo de macro e micronutrientes foi fornecido principalmente pela alimentação, com exceção da ingestão de vitamina D e E (tabela 5).

Segundo a Sociedade Brasileira de Medicina do Esporte ${ }^{15}$ a recomendação de energia para atletas deve corresponder de 37 a 41 kcal $/ \mathrm{kg} / \mathrm{dia}$. No presente estudo, os atletas do sexo masculino apresentaram consumo de $54,2 \mathrm{kcal} / \mathrm{kg} / \mathrm{dia}$ e as do sexo feminino de 50,1 kcal/kg/dia, indicando a necessidade do ajuste do aporte calórico para essa população.

A dieta dos atletas em estudo apresentou-se hipoglicídica ( $49,8 \pm 7,3 \%$ do VCT) e hiperproteica $(27,1 \pm 5,3 \%$ do VCT), utilizando-se como referência as recomendações da Sociedade Brasileira de Medicina do Esporte $^{15}$, que preconiza a ingestão de 60 a $70 \%$ de carboidratos e de 15 a 20\% de proteínas (tabela 6).

As maiores prevalências de inadequação do consumo de vitaminas e minerais foram para vitamina $B_{9}$, iodo e cálcio para os atletas do sexo masculino e vitamina $B_{9}$ cálcio e iodo para o sexo feminino (tabela 7).

Tabela 3. Distribuição, em média e desvio padrão, das variáveis antropométricas dos atletas em estudo de acordo com o sexo. São Paulo, 2013.

\begin{tabular}{c|c|c}
\hline Variável & Sexo masculino & Sexo feminino \\
\hline Peso $(\mathrm{kg})$ & $73,9 \pm 5,1$ & $60,5 \pm 6,6$ \\
\hline Estatura $(\mathrm{m})$ & $1,8 \pm 0,1$ & $1,7 \pm 0,1$ \\
\hline Indice de massa corporal $\left(\mathrm{kg} / \mathrm{m}^{2}\right)$ & $23,1 \pm 1,9$ & $21,2 \pm 1,4$ \\
\hline Dobras cutâneas de tríceps $(\mathrm{mm})$ & $7,4 \pm 1,8$ & $11,0 \pm 3,4$ \\
\hline Dobras cutâneas de bíceps $(\mathrm{mm})$ & $4,4 \pm 1,6$ & $8,7 \pm 1,3$ \\
\hline Dobras cutâneas subescapular $(\mathrm{mm})$ & $9,0 \pm 1,3$ & $9,6 \pm 1,1$ \\
\hline Dobras cutâneas torácica $(\mathrm{mm})$ & $4,3 \pm 1,2$ & $5,3 \pm 1,0$ \\
\hline Dobras cutâneas suprailíaca $(\mathrm{mm})$ & $8,3 \pm 1,3$ & $8,9 \pm 3,3$ \\
\hline Dobras cutâneas abdominal $(\mathrm{DCA})(\mathrm{mm})$ & $7,5 \pm 0,9$ & $11,7 \pm 5,3$ \\
\hline Dobras cutâneas de panturrilha medial $(\mathrm{mm})$ & $6,5 \pm 2,0$ & $10,1 \pm 1,7$ \\
\hline Circunferências de cintura $(\mathrm{cm})$ & $76,4 \pm 3,4$ & $69,9 \pm 3,7$ \\
\hline Circunferências de braço $(\mathrm{cm})$ & $32,4 \pm 1,3$ & $29,9 \pm 1,5$ \\
\hline Clrcunferência muscular do braço $(\mathrm{cm})$ & $30,1 \pm 1,3$ & $26,4 \pm 1,6$ \\
\hline Area muscular do braço corrigida $\left(\mathrm{cm}{ }^{2}\right)$ & $62,4 \pm 6,1$ & $49,2 \pm 6,7$ \\
\hline Área de gordura do braço $\left(\mathrm{cm}{ }^{2}\right)$ & $10,6 \pm 2,4$ & $13,4 \pm 3,5$ \\
\hline \%G (dobras cutâneas) & $10,8 \pm 2,7$ & $18,2 \pm 3,1$ \\
\hline \%G (BIA) & $9,8 \pm 2,6$ & $17,6 \pm 1,4$ \\
\hline
\end{tabular}

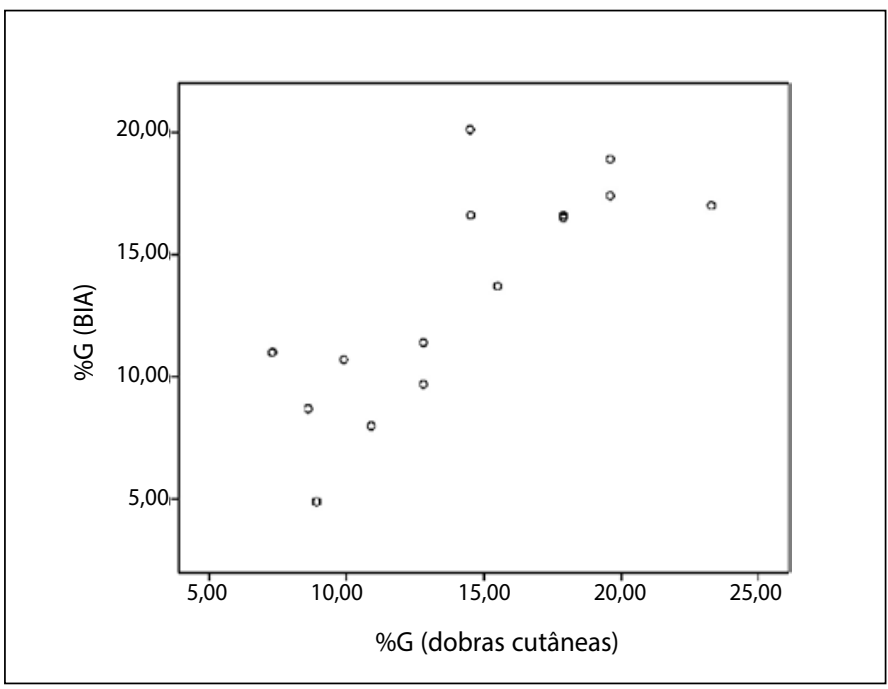

Figura 1. Correlação entre o percentual de gordura corporal obtido pelas dobras cutâneas e pela BIA. São Paulo, 2013.
Tabela 4. Suplemento alimentar consumido pelos atletas em estudo. São Paulo, 2013.

\begin{tabular}{c|c|c}
\hline Suplemento alimentar & $\mathbf{N}^{*}$ & $\%$ \\
\hline Endurox (Dextrose) & 13 & 86,7 \\
\hline Waxy Maize (Amilopectina) & 11 & 73,3 \\
\hline Accelerade (Repositor Energético) & 9 & 60,0 \\
\hline Creatina & 9 & 60,0 \\
\hline Iso WPI (Proteína do Soro Isolada) & 9 & 60,0 \\
\hline BCAA (Aminoácidos de Cadeia Ramificada) & 8 & 53,3 \\
\hline Beta-alanina & 7 & 46,7 \\
\hline D-Ribose & 6 & 40,0 \\
\hline Ômega-3 & 4 & 26,7 \\
\hline Maltodextrina & 3 & 20,0 \\
\hline Pharmaton ${ }^{\circledR}$ (Ginseng G115) & 3 & 20,0 \\
\hline Bicarbonato de sódio & 2 & 13,3 \\
\hline Nitro-Peak (Hidrolisado de Proteínas do Soro e Glutamina) & 2 & 13,3 \\
\hline Targifor C ${ }^{\oplus}$ (Ácido ascórbico) & 2 & 13,3 \\
\hline Carbonato de cálcio & 1 & 6,7 \\
\hline L-arginina & 1 & 6,7 \\
\hline Whey Protein (Proteína do Soro e Caseína) & 1 & 6,7 \\
\hline
\end{tabular}

*Resposta mútipla.

Tabela 5. Distribuição do consumo diário médio de macro e micronutrientes, provenientes pela alimentação e por suplementação, dos atletas em estudo. São Paulo, 2013.

\begin{tabular}{c|c|c|c|c|c|c}
\hline Nutriente & Alimentação & $\%$ & Suplementação & $\%$ & Total & $\%$ \\
\hline Carboidrato $(\mathrm{g})$ & 327,4 & 76,1 & 102,6 & 23,9 & 430,0 & 100,0 \\
\hline Proteína $(\mathrm{g})$ & 189,1 & 78,9 & 50,5 & 21,1 & 239,6 & 100,0 \\
\hline Lipídeo $(\mathrm{g})$ & 90,3 & 97,1 & 2,7 & 2,9 & 93,0 & 100,0 \\
\hline Vitamina $\mathrm{A}(\mu \mathrm{g})$ & 631,4 & 74,4 & 217,6 & 25,6 & 849,0 & 100,0 \\
\hline Vitamina $\mathrm{B}_{1}(\mathrm{mg})$ & 1,4 & 77,4 & 0,4 & 22,6 & 1,8 & 100,0 \\
\hline Vitamina $\mathrm{B}_{2}(\mathrm{mg})$ & 1,7 & 79,2 & 0,4 & 20,8 & 2,1 & 100,0 \\
\hline Vitamina $\mathrm{B}_{3}(\mathrm{mg})$ & 50,3 & 97,0 & 1,5 & 3,0 & 51,8 & 100,0 \\
\hline Vitamina $\mathrm{B}_{5}(\mathrm{mg})$ & 4,0 & 89,4 & 0,5 & 10,6 & 4,5 & 100,0 \\
\hline Vitamina $\mathrm{B}_{6}(\mathrm{mg})$ & 2,4 & 66,8 & 1,2 & 33,2 & 3,6 & 100,0 \\
\hline Vitamina $\mathrm{B}_{9}(\mu \mathrm{g})$ & 92,6 & 68,3 & 42,9 & 31,7 & 135,5 & 100,0 \\
\hline Vitamina $\mathrm{B}_{12}(\mu \mathrm{g})$ & 4,5 & 91,4 & 0,4 & 8,6 & 4,9 & 100,0 \\
\hline Vitamina $\mathrm{C}(\mathrm{mg})$ & 239,9 & 51,2 & 228,7 & 48,8 & 468,6 & 100,0 \\
\hline Vitamina $\mathrm{D}(\mu \mathrm{g})$ & 2,9 & 0,2 & 1600,5 & 99,8 & 1603,4 & 100,0 \\
\hline Vitamina $\mathrm{E}(\mathrm{mg})$ & 13,1 & 38,6 & 20,8 & 61,4 & 33,9 & 100,0 \\
\hline Cálcio $(\mathrm{mg})$ & 852,9 & 73,6 & 306,4 & 26,4 & 1159,3 & 100,0 \\
\hline Cobre $(\mu \mathrm{g})$ & 1284,0 & 72,5 & 486,4 & 27,5 & 1771,4 & 100,0 \\
\hline Ferro $(\mathrm{mg})$ & 16,0 & 82,8 & 3,3 & 17,2 & 19,3 & 100,0 \\
\hline Fósforo $(\mathrm{mg})$ & 1775,2 & 98,1 & 34,0 & 1,9 & 1809,2 & 100,0 \\
\hline lodo $(\mathrm{mg})$ & 79,7 & 100,0 & - & - & 79,7 & 100,0 \\
\hline Magnésio $(\mathrm{mg})$ & 312,0 & 56,3 & 242,1 & 43,7 & 554,1 & 100,0 \\
\hline Manganês $(\mathrm{mg})$ & 1,8 & 71,4 & 0,7 & 28,6 & 2,5 & 100,0 \\
\hline Potássio $(\mathrm{mg})$ & 3161,7 & 98,7 & 40,3 & 1,3 & 3202,0 & 100,0 \\
\hline Selênio $(\mu \mathrm{g})$ & 107,7 & 89,0 & 13,3 & 11,0 & 121,0 & 100,0 \\
\hline Sódio $(\mathrm{mg})$ & 3567,4 & 87,7 & 500,1 & 12,3 & 4067,5 & 100,0 \\
\hline Zinco $(\mathrm{mg})$ & 19,0 & 95,5 & 0,9 & 4,5 & 19,9 & 100,0 \\
\hline & & & & &
\end{tabular}

Tabela 6. Consumo de macronutrientes pelos atletas em estudo. São Paulo, 2013.

\begin{tabular}{c|c|c|c|c|c|c}
\hline \multirow{2}{*}{ Consumo } & \multicolumn{6}{|c}{ Nutriente } \\
\cline { 2 - 7 } & \multicolumn{2}{|c|}{ Carboidrato } & \multicolumn{2}{|c}{ Proteína } & \multicolumn{2}{c}{ Lipídeo } \\
\cline { 2 - 7 } & $\mathbf{n}$ & $\%$ & $\mathbf{n}$ & $\%$ & $\mathbf{n}$ & $\%$ \\
\hline Baixo & 14 & 93,3 & - & - & 5 & 33,3 \\
\hline Adequado & 1 & 6,7 & 1 & 6,7 & 8 & 53,4 \\
\hline Elevado & - & - & 14 & 93,3 & 2 & 13,3 \\
\hline Total & 15 & 100,0 & 15 & 100,0 & 15 & 100,0 \\
\hline
\end{tabular}


Tabela 7. Prevalência de inadequação do consumo de micronutrientes dos atletas em estudo. São Paulo, 2013.

\begin{tabular}{|c|c|c|c|}
\hline \multirow{2}{*}{ Nutriente } & \multicolumn{3}{|c|}{ Prevalência de inadequação (\%) } \\
\hline & Sexo masculino & Sexo feminino & Ambos os sexos \\
\hline Vitamina A & 29,1 & 29,1 & 29,1 \\
\hline Vitamina $\mathrm{B}_{1}$ & 27,4 & 15,9 & 22,0 \\
\hline Vitamina $\mathrm{B}_{2}$ & 17,1 & 6,1 & 11,9 \\
\hline Vitamina $\mathrm{B}_{3}$ & 4,0 & 0,1 & 2,1 \\
\hline Vitamina $B_{5}$ & * & * & * \\
\hline Vitamina $B_{6}$ & 14,7 & 3,2 & 9,3 \\
\hline Vitamina $\mathrm{B}_{9}$ & 97,1 & 99,9 & 98,5 \\
\hline Vitamina $\mathrm{B}_{12}$ & 12,5 & 17,1 & 14,7 \\
\hline Vitamina C & 10,6 & 12,5 & 11,5 \\
\hline Vitamina D & 29,1 & 34,5 & 31,6 \\
\hline Vitamina E & 3,6 & 12,5 & 7,8 \\
\hline Cálcio & 34,5 & 61,8 & 47,2 \\
\hline Cobre & 8,1 & 14,7 & 11,2 \\
\hline Ferro & 0,8 & 2,3 & 1,5 \\
\hline Fósforo & 3,2 & 13,6 & 8,1 \\
\hline lodo & 65,5 & 56,0 & 61,1 \\
\hline Magnésio & 0,2 & 8,1 & 3,9 \\
\hline Manganês & * & * & * \\
\hline Potássio & * & * & * \\
\hline Selênio & 13,6 & 8,9 & 11,4 \\
\hline Sódio & * & * & * \\
\hline Zinco & 3,2 & 6,1 & 4,5 \\
\hline
\end{tabular}

*apenas adequate intake (Al) disponível.

\section{DISCUSSÃO}

Identificou-se, no presente estudo, que os atletas apresentaram percentual de gordura baixo ou adequado, sem risco para o desenvolvimento de alterações metabólicas.

Quanto às variáveis peso e altura foram encontrados valores semeIhantes por Nagaoka et al. ${ }^{22}$ para o sexo feminino, sendo a média de

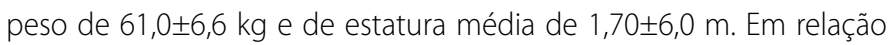
ao sexo masculino foram encontrados valores ligeiramente superiores,

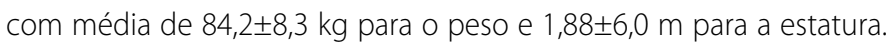
Contudo, essas diferenças podem ser explicadas pelo fato desses autores terem incluso adultos em seu estudo.

Quanto às DCT, DCSE, DCSI, DCA, o presente estudo identificou medidas semelhantes as de Schneider e Meyer ${ }^{23}$ para DCT $(11,3 \pm 3,3$ $\mathrm{mm})$ e DCSE $(9,7 \pm 4,8 \mathrm{~mm})$ para o sexo feminino, sendo que DCSI e DCA apresentaram-se maiores no estudo desses autores $(12,2 \pm 5,0$ e $14,9 \pm 5,2$, respectivamente). Para o sexo masculino foram encontrados valores superiores ao do presente estudo para DCT $(8,4 \pm 2,8 \mathrm{~mm}) \mathrm{e}$ DCA $(10,7 \pm 4,0 \mathrm{~mm})$. O valor da DCSE apresentou-se menor $(7,4 \pm 2,4$

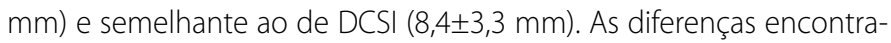
das podem ser explicadas pela faixa etária dos atletas em estudo, que estavam na fase púbere.

Referente à CC, os valores encontrados de $76,4 \pm 3,4 \mathrm{~cm}$ para o sexo

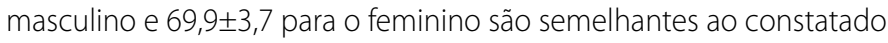
por Meliscki, Monteiro e Giglio ${ }^{24}$, ao avaliarem a relação entre dor e treinamento em nadadores de elite entre 13 e 28 anos, que apresentaram CC correspondente a $76,4 \pm 3,8 \mathrm{~cm}$ para o sexo masculino e $67,3 \pm 4,6$ para o feminino, sendo considerado como CC o ponto médio entre a crista ilíaca e o último arco costal.

Quanto ao percentual de gordura, no estudo de Carvalho et al. ${ }^{25}$, que avaliaram os indicadores antropométricos e dietéticos do estado nutricional de adolescentes nadadores competitivos de 10 a 19 anos do Rio de Janeiro, Brasil, encontrou-se valores de 7,9 $\pm 2,8 \%$ para o sexo masculino e $17,4 \pm 4,3 \%$ para o feminino, sendo a diferença explicada provavelmente pela fórmula utilizada e pela faixa etária mais abrangente.

Os atletas do presente estudo apresentaram alto consumo de suplementos alimentares, que é amplamente difundido em adolescentes, sobretudo aos atletas e frequentadores de academia ${ }^{26}$. Contudo, diferentemente do presente estudo, onde se identificou maior utilização de suplementos energéticos, outros estudos indicam o maior consumo de suplementos proteicos 27,28 .

Embora os suplementos mais utilizados pelos atletas do presente estudo sejam os energéticos, os mesmos apresentaram alto consumo de proteínas por meio da dieta. Tal fato é corroborado por outros estudos que verificaram baixo consumo de carboidratos e ingestão elevada de proteínas em atletas, independentemente do esporte praticado, o que pode causar prejuízos no desempenho, como fadiga precoce e aumento de recuperação pós-treino, uma vez que esse macronutriente caracteriza-se como a principal fonte de energia 29,30 .

O consumo adequado de carboidratos está relacionado também com a prevenção das infecções de vias aéreas superiores, que são frequentes em nadadores. Ramos et al. ${ }^{31}$ verificaram que a ingestão recomendada desse macronutriente promoveu diminuição dos sintomas dessas infecções, ao passo que o consumo inadequado relacionou-se com o surgimento dos mesmos. A suplementação com carboidratos no pré-treino também se mostrou eficaz ao sistema imunológico, promovendo menores concentrações de cortisol e menor perturbação da contagem total de leucócitos e suas subclasses, de acordo com Mendes et al. ${ }^{32}$.

A ingestão inadequada de vitaminas e minerais também pode levar a prejuízos no desempenho esportivo. Em estudo realizado por Leal et al. ${ }^{33}$, que objetivou avaliar o padrão de consumo alimentar de adolescentes verificou-se uma inadequação de ingestão de cálcio de $90,0 \%$ para o sexo masculino e $96,0 \%$ para o sexo feminino, dado maior do que o observado no presente estudo.

Na adolescência, o consumo de cálcio adequado é fundamental, tendo em vista que o acúmulo de massa óssea ocorre desde o início da puberdade até a segunda década de vida. Um consumo insuficiente desse mineral contribui para a elevação dos níveis de $1,25(\mathrm{OH})_{2} \mathrm{D}_{3}$ e paratormônio, promovendo o influxo de cálcio no adipócito e consequente aumento da lipogênese e redução da lipólise ${ }^{34}$.

A adoção de uma alimentação diversificada, com consumo de leites e derivados, é capaz de atender a necessidade de cálcio, assim como de outros nutrientes. Mensagens de educação nutricional que enfatizem melhor conhecimento sobre a importância do consumo de cálcio para a saúde e a criação de políticas públicas que favoreçam a fortificação de alimentos com esse mineral são estratégias que podem ser utilizadas para maximizar a sua ingestão ${ }^{35}$.

Vitolo et al..$^{36}$ verificaram que $89 \%$ dos adolescentes da cidade de São Leopoldo, RS, Brasil, apresentaram ingestão insuficiente de vitamina $B_{9}$, sendo que os atletas do presente estudo apresentaram prevalência de inadequação superior. O consumo deficiente da vitamina relaciona-se ao aumento do estresse oxidativo, que é frequente em nadadores, destacando-se a necessidade de programas de educação nutricional a fim de aumentar o consumo de alimentos fontes de vitamina $\mathrm{B}_{9}{ }^{37}$.

Não foram encontrados estudos que avaliaram o consumo ou os níveis séricos de iodo em adolescentes, indicando a necessidade dessa mensuração.

Deve-se considerar, ao se avaliar o consumo alimentar de quaisquer populações, o erro intrínseco ao programa utilizado, que pode sub ou superestimar a ingestão de macro e micronutrientes. Para a análise de micronutrientes, deve-se considerar também que o recomendado para a população em geral não se aplica a atletas, sugerindo-se que as necessidades de vitaminas e minerais sejam maiores nesse grupo. 


\section{CONCLUSÃO}

Conclui-se que os atletas do presente estudo apresentaram estado nutricional adequado, fundamental para o desempenho esportivo. Observou-se uso excessivo de suplementos alimentares, consumo de energia e de proteínas elevado e baixo consumo de carboidratos. As maiores prevalências de inadequações para a ingestão de micronu- trientes foram para vitamina $B_{9}$, cálcio e iodo, indicando necessidade de intervenção nutricional nesse grupo.

Todos os autores declararam não haver qualquer potencial conflito de interesses referente a este artigo.

\section{REFERÊNCIAS}

1. Prestes J, Leite RD, Leite GS, Donatto FF, Urtado CB, Bartolomeu Neto J, et al. Características antropométricas de jovens nadadores brasileiros do sexo masculino e feminino em diferentes categorias competitivas. Rev Bras Cineantropom Desempenho Hum. 2006;8:25-31.

2. Perez AJ, Bassini CF, Pereira BMF, Sarro KJ. Correlação entre variáveis antropométricas e o comprimento e a frequência da braçada de nadadores do Espírito Santo Rev Mackenzie Educ Fís Esporte. 2011;10:19-27.

3. Lagacione AC, Pereira GG, Tumelero S, Guilherme C. Importância da alimentação e hidratação para jovens atletas. Revisão de literatura. Lect Educ Fís Deportes. 2012;17:1.

4. Nacif M, Viebig RF. Avaliação antropométrica nos ciclos da vida: uma visão prática. 2.ed. São Paulo: Metha, 2011.

5. Kabasakalis A, Kalitsis K, Tsalis G, Mougios V. Imbalanced nutrition of top-level swimmers. Int J Sports Med. 2007;28:780-6.

6. Prado ES, Barroso SS, Góis HO, Reinert T. Estado de hidratação em nadadores após três diferentes formas de reposição hídrica na cidade de Aracaju, SE. Fit Perf J. 2009:8:218-25.

7. Rankin JW. Dietary carbohydrate and performance of brief, intense exercise. Sports Sci Exchange. 2000;13:1.

8. World Health Organization. Development of a WHO growth reference for school-aged children and adolescents. Geneva: WHO, 2007

9. Slaughter MH, Lohman TG, Boileau RA, Horswill CA, Stillman RJ, Van Loan MD, et al. Skinfold equations for estimation of body fatness in children and youth. Hum Biol. 1988;60:709-23.

10. Jackson AS, Pollock M. Practical assessment of body composition. Physi Sportsmed. 1985;13:76-90.

11. Deurenberg P, Kusters CSL, Smit HE. Assessment of body composition by bioeletrical impedance in children and young adults is strongly age-dependent. Eur J Clin Nutr. 1990;44:261-8.

12. Frisancho AR. Anthropometric standards for the assessment of growth and nutritional status. Michigan: The University of Michigan Press, 1990.

13. Freedman DS, Serdula MK, Srinivasan SR, Berenson GS. Relation of circumferences and skinfold thicknesses to lipid and insulin concentrations in children and adolescents: the Bogalusa Heart Study. Am J Clin Nutr. 1999;69:308-17.

14. World Health Organization. Obesity: preventing and managing the global epidemic. Geneva:WHO, 1998.

15. Hernandez AJ, Nahas RM. Modificaçōes dietéticas, reposição hídrica, suplementos alimentares e drogas: comprovação de ação ergogênica e potenciais riscos para a saúde. Rev Bras Med Esporte. 2009;15:2-12.

16. Institute of Medicine. Dietary Reference Intakes for calcium, phosphorus, magnesium, vitamin $D$, and fluoride. Washington: National Academies Press, 1997.

17. Institute of Medicine. Dietary Reference Intakes for thiamin, riboflavin, niacin, vitamin B6, folate, vitamin B12, pantothenic acid, boitin and choline. Washington: National Academies Press, 1998.

18. Institute of Medicine. Dietary Reference Intakes for vitamin A, vitamin K, arsenic, boron, chromium, copper, iodine, iron, manganese, molybdenum, nickel, silicon, vanadium, and zinc. Washington: $\mathrm{Na}$ tional Academies Press, 2000.

19. Institute of Medicine. Dietary Reference Intakes for vitamin C, vitamin E, selenium, and carotenoids. Washington: National Academies Press, 2000
20. Institute of Medicine. Dietary Reference Intakes for water, potassium, sodium, chloride, and sulfate. Washington: The National Academies Press, 2004.

21. Institute of Medicine. Dietary Reference Intakes for calcium and vitamin D. Washington: The National Academies Press, 2011.

22. Nagaoka AM, Yoshimura MT, Marques SH, Frutuoso MFP, Mendes Netto RS. Perfil antropométrico de nadadores de alto nível. Rev Bras Nutr Esportiva. 2008;2:374-80.

23. Schneider $P$, Meyer F. Avaliação antropométrica e da força muscular em nadadores pré-púberes e púberes. Rev Bras Med Esporte. 2005;11:209-13.

24. Meliscki GA, Monteiro LZ, Giglio CA. Associação entre dor e treinamento em nadadores de elite. Rev Bras Prom Saúde. 2011;24:116-22.

25. Carvalho FG, Rosa FT, Suen VMM, Freitas EC, Padovan GJ, Marchini JS. Evidence of zinc deficiency in competitive swimmers. Nutrition. 2012;28:1127-31.

26. Alves C, Lima RVB. Uso de suplementos alimentares por atletas. J Pediatr. 2009;85:287-94.

27. Albuquerque MM. Avaliação do consumo de suplementos alimentares nas academias de Guará-DF. Rev Bras Nutr Esportiva. 2012;6:112-7.

28. Zilch MC, Soares BM, Bennemann GD, Sanches FFZ, Cavazzotto TG, Santos EF. Análise da ingestão de proteínas e suplementação por praticantes de musculação nas academias centrais da cidade de Guarapuava-PR. Rev Bras Nutr Esportiva. 2012;6:381-8.

29. Flores TG, Mattos KM. Análise de macronutrientes e índice glicêmico consumidos nas refeições antes, durante e após o treino por atletas de futebol profissional de Camaquã-RS. Rev Bras Nutr Esportiva. 2011;5:394-401.

30. Coelho B, Azeredo C, Bressan E, Gandelini J, Gerbelli N, Cavignato P. Perfil nutricional e análise comparativa dos hábitos alimentares e estado nutricional de atletas profissionais de basquete, karatê, tênis de mesa e voleibol. Rev Bras Nutr Esportiva. 2009;3:570-7.

31. Ramos DD, Toriani S, Silva S, Dalquano EC. Avaliação nutricional e sintomas metabólicos de nadadores de competição. Rev Bras Nutr Esportiva. 2010;4:217-24.

32. Mendes EL, Brito CJ, Batista ES, Silva CHO, Paula SOP, Natali AJ. Influência da suplementação de car boidrato na função imune de judocas durante o treinamento. Rev Bras Med Esporte. 2009;15:58-61.

33. Leal GVS, Philippi ST, Matsudo MM, Toassa EC. Consumo alimentar e padrão de refeições de adolescentes, São Paulo. Rev Bras Epidemiol. 2010;13:457-67.

34. Santos LC, Martini LA, Freitas SN, Cintra IP. Ingestão de cálcio e indicadores antropométricos entre adolescentes. Rev Nutr. 2007;20:275-83.

35. Pereira GAP, Genaro OS, Pinheiro MM, Szejnfeld VL, Martini LA. Cálcio dietético - estratégias para otimizar o consumo. Rev Bras Reumatol. 2009;49:164-80

36. Vitolo MR, Canal Q, Campagnolo PDB, Gama CM. Fatores associados ao risco de ingestão insuficiente de folato entre adolescentes. J Pediatr. 2006;82:121-6.

37. Uehara SK, Rosa G. Associação da deficiência de ácido fólico com alterações patológicas e estratégias para sua prevenção: uma visão crítica. Rev Nutr. 2010;23:881-94. 